\title{
A educação física escolar como agente da prevenção e do tratamento do sobrepeso e obesidade infantil*
}

\section{The physical education as agent of prevention and tratment from childhood obesity and overweight}

Tiago Souza de Menezes ${ }^{1}$ Renata Aparecida Elias Dantas ${ }^{2}$
Recebido em: 20/09/2016. Aprovado em: 29/11/2016.

1 Graduando - Centro Universitário de Brasília - UniCEUB - Brasília-DF - Brasil. Email: tiago_menezes95@hotmail.com.

2 Professora Doutora - Centro Universitário de Brasília - UniCEUB - Brasília-DF - Brasil. Email: profrenataelias@yahoo.com.br.

\section{Resumo}

Este estudo analisou se as aulas contribuem para prevenção da obesidade infantil e o Índice de Massa Corporal (IMC) dos alunos. A amostra foi constituída por 44 crianças e um professor. Verificou-se o IMC para classificação da obesidade e aplicado um questionário para o professor para avaliar o conhecimento do mesmo sobre o assunto obesidade. Foram observadas aulas para verificar se este executava alguma ação para tratar o quadro de obesidade de seus alunos. 65,9\% das crianças foram classificadas como eutróficas, enquanto $34,1 \%$ apresentavam sobrepeso ou obesidade. Não houve diferença entre meninos e meninas na massa corporal $(\mathrm{p}=0,523)$, na estatura $(\mathrm{p}=0,352)$ e no IMC $(\mathrm{p}=$ $0,897)$. O professor avaliado abordou que a intervenção feita por ele é, apenas, uma conscientização dos males causados pela obesidade. As intervenções realizadas pelo professor nas aulas não eram efetivas para combate ou prevenção da obesidade, devido à falta de aulas disponíveis para um trabalho dessa proporção.

Palavras-chave: Sobrepeso. Obesidade Infantil. Educação Física Escolar.

\begin{abstract}
This study examined if the teacher made some intervention on the class to prevent childhood obesity. The sample was built by 44 children and one teacher. Was measured the BMI, to rating the children of obesity and applied a questionnaire to the teacher to assess his knowledge about the subject obesity. Were observed classes to verify if the teacher performs some activity to deal with the status of obesity of students. $65.9 \%$ from the children were eutrophic, while $34.1 \%$ were overweight or obesity. Did not has difference between boys and girls in body mass $(\mathrm{p}=0.523)$, in height $(\mathrm{p}=0.352)$ and BMI $(\mathrm{p}=0.897)$. The teacher evaluated addressed that the intervention made by him, it is only an awareness about the illnesses, caused by obesity. The interventions made by the teacher in the classes were not effective in fighting against overweight and obesity, due a lack of classes available to a program with this proportion.
\end{abstract}

Keywords: Overweight. Childhood Obesity. Scholar Physical Education. 


\section{Introdução}

A obesidade é caracterizada por ser uma doença crônica pelo aumento excessivo de tecido adiposo, por meio de desequilíbrio energético, influenciado por diversos fatores, incluindo fatores comportamentais, genéticos, endócrinos, farmacológicos e nutricionais, contribuindo para um impacto negativo na saúde, aumentando índices de Diabetes Tipo 2 e outras doenças crônicas (DIAS; MONTENEGRO; MONTEIRO, 2014).

No entanto, dados indicam que crianças reduzem seus hábitos de realizar atividade física, com o tempo em que vão envelhecendo, e de fato, essas reduções aumentam o risco de obesidade e outras doenças crônicas associadas a ela (WOLFF-HUGHES et al., 2014). Assim, de acordo com Kwon et.al. (2015), uma vida ativa, juntamente com uma boa alimentação por toda a infância e adolescência é possível prevenir o desenvolvimento da obesidade na vida adulta, tendo em vista que esses hábitos são adquiridos das famílias e escolas.

Segundo a Lei de Diretrizes e Bases da Educação (LDB) (1996), a educação física faz parte da proposta pedagógica da escola no currículo de educação básica, sendo um componente obrigatório deste âmbito escolar (BRASIL, 1996).

Para Ennis (2013), atualmente, a sociedade, o governo, até mesmo as escola, não possuem o reconhecimento de que a saúde está, relativamente, relacionada com exercício físico. Dessa maneira, a sociedade acaba dando uma maior ênfase no quesito mente do que corpo, implantando um projeto educacional no qual prioriza matérias como matemática, história, ciências, filosofia, mais do que a ciência do corpo humano. Esse é um fato comum que vem ocorrendo em vários países, que, frequentemente, negligenciam o prazer da ciência associada ao desempenho físico.

Assim sendo, a cada dia, observa-se o aumento da obesidade infantil, em virtude de as crianças estarem cada vez mais sedentários e menos ativos de acordo com padrão social no qual estão inseridos. Por isso, cresce-se a necessidade de se trabalhar de forma interdisciplinar entre as aulas de Educação Física, a parte nutricional uma alimentação adequada para incentivar uma vida longilínea e saudável, sendo assim, uma questão educacional, de responsabilidade da família e da escola (SANTOS et al., 2007).
Em escolas infantis da Itália e do Brasil, aulas de educação física chegam a ser ministradas por professores que não são especialistas nessa área de atuação. Consequentemente, desse modo, a educação física se torna, totalmente, negligenciada, variando de escola para escola, dependendo o currículo do professor de dentro da escola (SACCHETTI et al., 2013).

De acordo com a literatura, existe a necessidade de futuras de intervenções na comunidade, com intuito de desenvolver uma melhor qualidade de vida de adolescentes e por meio de uma maneira em que tais mudanças venham a permanecer na vida adulta desses adolescentes. Por esse motivo, quanto mais conhecimento conseguir ser agregado sobre comportamentos sedentários, regularidade na prática de exercício físico, dados antropométricos, perfil lipídico, hábitos alimentares, uma maior contribuição e incentivo será dado para essa população (GUIMARÃES et al., 2013).

Conforme for o nível de obesidade encontrada nas crianças, é necessário realizar programas lúdicos, que possam prover diversas intensidades envolvendo exercícios aeróbicos com características de resistência. Dessa maneira, é possível proporcionar um desenvolvimento tanto psicológico, como fisiológico e acadêmico (ESMERALDO et al., 2014).

O objetivo desse estudo foi analisar se a aula feita do professor de Educação Física contribui para prevenção da obesidade infantil. Além disso, descrever o Índice de Massa Corporal (IMC) dos alunos da turma do professor participante do estudo.

\section{Material e Métodos}

\subsection{Natureza do estudo}

Essa pesquisa foi um estudo transversal de caráter exploratório, foi aprovado pelo comitê de ética e pesquisa do Centro Universitário de Brasília, seguindo as diretrizes éticas nacionais, da resolução 466/12 CMS/MS, o CAAE: 51119415.0.0000.0023 e o número do parecer: 1.372.675.

\subsection{Participantes}

A amostra foi constituída por um professor de educação física, e por 44 alunos de sua turma do Ensino Fundamental I de ambos os sexos, com idade entre 8 e 9 anos, de uma Escola Particular. 


\subsection{Instrumentos e procedimentos}

Para a mensuração do IMC (Índice de Massa Corporal), foi usado o peso (em quilogramas) divido pela estatura (em metros) ao quadrado de acordo com World Health Organization (2007). Para ser adquirido o peso necessário para realização do calculo IMC, foi utilizado uma balança digital do modelo Techline. No entanto, foi utilizado o estadiômetro portátil com escala metálica do modelo Sanny para mensuração da estatura. Para mensuração das crianças, foi necessário estarem sem tênis e com roupas leves.

Foi aplicado um questionário com oito perguntas abertas formulado por Nascimento (2013), para analisar a percepção do professor em relação a obesidade e também identificar se tal professor utiliza alguma metodologia para combater o sobrepeso e obesidade infantil.

Foram observadas três aulas propostas pelo professor, para analisar se estas apresentavam algum tipo de intervenção para prevenção ou conscientização dos alunos para o problema da obesidade.

\subsection{Analise dos dados}

A análise descritiva foi utilizada para calcular a média e o desvio padrão de todas as variáveis. A normalidade dos dados foi verificada pelo teste de Shapiro-Wilk e a estatística paramétrica foi adotada. A análise de frequência foi utilizada para a caracterização para caracterização da amostra. Possíveis diferenças entre os sexos foram analisadas pelo Teste $\mathrm{T}$ independente. Todas as análises estatísticas foram realizadas no software estatístico SPSS versão 21.0 (SPSS Inc., Somers, NY, USA). Adotou-se $\mathrm{p} \leq 0,05$ como nível de significância.

\section{Resultados e Discussão}

Observou-se que 65,9\% (n-29) das crianças eram eutróficas, enquanto $34,1 \%$ (15) apresentavam sobrepeso ou obesidade. Estratificando por gênero, 66,7\% (12) dos meninos eram eutróficos, enquanto 33,3\% (6) apresentavam sobrepeso e obesidade. $65,4 \%$ (17) das meninas eram eutróficas, enquanto $34,6 \%$ (9) apresentavam sobrepeso e obesidade. As características da amostra estão expostas na tabela 1. Não houve diferença entre meninos e meninas na massa corporal $(\mathrm{p}=0,523)$, na estatura $(\mathrm{p}=0,352)$ e no $\operatorname{IMC}(\mathrm{p}=0,897)$.
Tabela 1 Características da amostra e significância entre gêneros.

\begin{tabular}{|c|c|c|c|c|c|}
\hline Variável & Média & DP & Mínimo & Máximo & $\mathrm{p}$ \\
\hline \multicolumn{6}{|c|}{ Massa Corporal (kg) } \\
\hline Meninos (18) & 31,27 & 5,08 & 24,20 & 44,00 & \multirow{2}{*}{0,523} \\
\hline Meninas (26) & 30,14 & 6,12 & 22,00 & 44,00 & \\
\hline \multicolumn{6}{|l|}{ Estatura (m) } \\
\hline Meninos (18) & 1,33 & 0,06 & 1,24 & 1,42 & \multirow{2}{*}{0,352} \\
\hline Meninas (26) & 1,32 & 0,07 & 1,22 & 1,48 & \\
\hline \multicolumn{6}{|l|}{ IMC $\left(\mathrm{kg} / \mathrm{m}^{2}\right)$} \\
\hline Meninos (18) & 17,48 & 2,01 & 14,54 & 22,14 & \multirow{2}{*}{0,897} \\
\hline Meninas (26) & 17,37 & 2,91 & 13,12 & 24,14 & \\
\hline
\end{tabular}

Os principais resultados deste estudo foram apresentados na Figura 1, que mostram a comparação da classificação do IMC dos alunos, em eutróficos, sobrepeso e obesos, divididos por gênero, de acordo com as normas de recomendação do World Health Organization (WHO) com a mensuração por meio do gênero e idade. Contudo, a presente amostra possui $33,33 \%$ do sexo masculino e $34,62 \%$ do sexo feminino foram caracterizados com sobrepeso ou obesidade.

Dessa maneira, os homens apresentaram $11,11 \% \mathrm{e}$ $22,22 \%$ com sobrepeso e obesidade respectivamente, enquanto as mulheres apresentaram 23,08\% e 11,54\% com sobrepeso e obesidade. Assim, os homens apresentaram $66,67 \%$ eutróficos, quando as mulheres resultaram em $65,38 \%$ eutróficas.

Figura 1 - Comparação da classificação do IMC de acordo com WHO

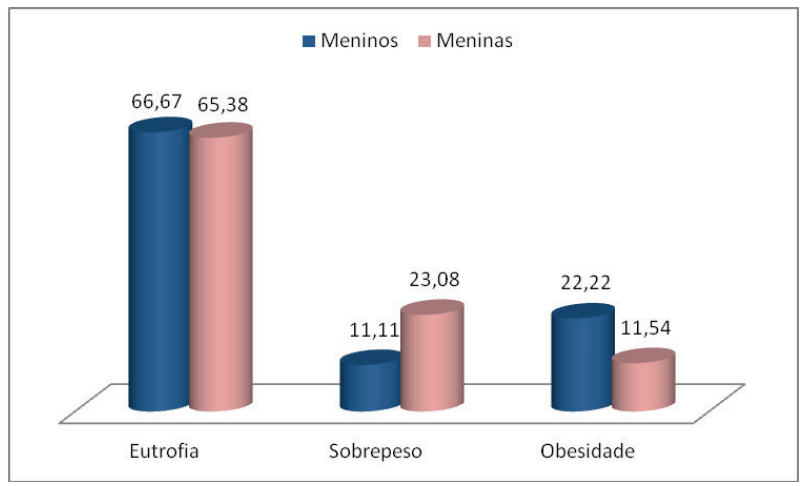

Fonte : Dos autores.

Quanto ao questionário aplicado (Anexo 1), foram extraídos os seguintes pontos de vista do professor: para a resposta da pergunta 5 foi obtido "O sedentarismo e a falta de disposição para a atividade física são fatores que interferem". A resposta obtida para pergunta 6 foi "As aulas de Educação Física estimulam a prática de exercício físico, diminuindo o risco de obesidade infantil". Enquanto a resposta da pergunta 7 foi "Realizando um trabalho 
educacional para conscientizar sobre os perigos da obesidade infantill". Para a resposta da pergunta 8 , foi obtida a afirmação "Com a presença do profissional de Educação Física os alunos praticarão os exercícios corretos".

O nível de sobrepeso e obesidade infantil nas escolas vem crescendo exponencialmente, por meio de hábitos sedentários inseridos no dia a dia das famílias, juntamente a maus hábitos alimentares, podendo gerar uma pobre saúde física, mental e social, dessa maneira vindo a deteriorar o quadro de qualidade de vida desses indivíduos (SACCHETTI et al., 2015; SBC, 2005).

Contudo, está sendo utilizado com maior frequência o IMC por idade, para classificar as crianças com idade entre 5 e 19 anos, com sobrepeso e obesidade, de acordo com World Health Organization (WHO), devido a sua ampla aplicabilidade e por ser, altamente, reconhecido por diversos países, na tentativa de avaliar a magnitude de problemas na saúde pública como a obesidade infantil (LEAO et al., 2003; WHO, 2007).

Em um estudo realizado por Ramos (2013), foi encontrado uma prevalência de sobrepeso e obesidade em crianças de 10 a 14 anos de $23,5 \%$ no sexo feminino e de $22,6 \%$ no sexo masculino, resultados inferiores aos do presente estudo em que foi encontrado $34,62 \%$ no sexo feminino e de $33,33 \%$ no sexo masculino, em crianças com idade entre 8 e 9 anos.

Uma pesquisa que avaliou 5235 crianças do Reino Unido com idade de 9 a 12 anos, 18,5\% (965) foram classificadas com sobrepeso, enquanto 4,5\% (234) com obesidade (LAWLOR et. al., 2010). No presente estudo, o público com sobrepeso foi similar a tal pesquisa com um valor de $18,18 \%$, portanto, em relação ao nível de obesidade, foi mais elevado, alcançando $15,91 \%$, sendo considerado bem superior.

No entanto, estudos de análise da obesidade infantil, comparando instituições públicas e privadas, sexo, faixa etária e regiões do Brasil e maturação sexual, resultaram em um maior índice de obesidade em instituições privadas comparado com as públicas. Não houve diferença significativa entre os sexos, faixa etária, regiões e maturação sexual quando a obesidade foi classificada por meio do IMC (RICARDO et al., 2009; RAMOS et al., 2013). Resultados similares ao do presente estudo, em referência ao sexo e à faixa etária, não apresentaram diferença significativa entre eles.

Em um estudo com o objetivo de identificar o conceito de saúde por meio das ideias de professores de educação física e sua consciência sobre Saúde Renovada, observou-se que todos os professores possuem ideias similares de saúde, relacionando-a com qualidade de vida. E, apesar de todos os professores trabalharem aspectos de saúde, principalmente na dimensão conceitual, nenhum dos professores apresentou conhecimento sobre Saúde Renovada (ZANCHA et al. 2013). Isto aconteceu, também, com esse estudo onde o professor mostrou, apenas, conscientizar as crianças sobre os malefícios da obesidade infantil, sem fazer um trabalho abordando a prática, justificando por não ser suficiente uma aula por semana para gerar adaptações que resultem em diminuição da obesidade.

Em estudo em que foram avaliadas as atividades em que as crianças obesas apresentam uma maior aderência, a atividade mais prazerosa foi a queimada (CELESTRINO; COSTA, 2006). É possível usar as aulas de educação física para relacionar os exercícios com maior aderência desses alunos, contudo, realizando adaptações das regras, com fim de gerar um maior gasto calórico nesses indivíduos.

Em um estudo realizado por Guedes e Guedes (2001), com o intuito de avaliar a intensidade das aulas de Educação Física Escolar em relação à promoção de saúde, foi apresentado que, em uma aula com duração de 50 minutos, foram disponibilizados, apenas, 37 a 40 minutos com atividades previstas pela aula. Portanto, o tempo disponibilizado para prática foi caracterizado com intensidades de curta duração e de baixa intensidade, juntamente a um currículo escolar no qual apresenta apenas uma hora de educação física escolar por semana, inviabilizando alcances de benefícios satisfatórios para promoção da saúde (GUEDES; GUEDES, 2001), o que, também, foi observado no presente estudo. Além disso, o temo efetivo das aulas assistidas foi menor ainda (entre 20 e 30 minutos).

Por outro lado, evidências mostram que programas de Educação Física, bem estruturados, realçam a efetividade das intervenções nas melhoras das habilidades e capacidades físicas, diminuindo as atividades sedentárias diárias e concomitantemente diminuindo o nível de sobrepeso e obesidade infantil, provendo, também, mudanças positivas nos hábitos das crianças (SACCHETTI et.al., 2013; SACCHETTI et.al., 2015).

Quando as respostas do questionário e a observação das aulas no presente estudo foram confrontadas, foi possível observar que o professor fez algumas inferências na aula para os alunos sobre obesidade infantil e seus ma- 
lefícios a saúde, porém, com abordagem conceitual em suas aulas. A parte prática da aula não teve como foco um trabalho para socialização.

Apesar de a maioria das crianças desse estudo serem eutróficas, $33,33 \%$ do sexo masculino e $34,62 \%$ do sexo feminino foram caracterizados com sobrepeso ou obesidade. Dessa forma, talvez fosse interessante proporcionar aulas práticas que também contribuíssem para a diminuição do sobrepeso e obesidade. Conforme exposto pelo professor esse aspecto se torna difícil quando se tem, apenas, uma aula na semana.

\section{Conclusão}

Apesar de a maioria das crianças serem eutróficas, muitos estavam com sobrepeso ou obesidade, dessa forma, seria valido uma maior preocupação por parte do professor quanto a uma intervenção para prevenção ou combate à obesidade.

Pode-se inferir que as intervenções realizadas nas aulas desse estudo não foram eficientes no combate ao sobrepeso e à obesidade. Isso ocorreu, também, devido à falta de mais aulas disponíveis para desenvolver um trabalho dessa proporção, visto que nessa escola as aulas aconteciam, somente, uma vez por semana.

Um trabalho conjunto que envolva pais, coordenadores pedagógicos e professores pode ajudar para que esse problema de saúde pública comece a ser trabalhado de forma mais eficiente.

Sugerem-se estudos que envolvam um trabalho multidisciplinar nas escolas para verificar se, dessa forma, a prevenção e o combate à obesidade seriam mais eficientes na infância.

\section{Referências}

BRASIL. Lei no 9.394 de 20 de dezembro de 1996. Estabelece as diretrizes e bases da educação nacional. Brasília, 1996. Disponível em: <http://www.planalto.gov.br/ ccivil_03/LEIS/19394.htm>. Acesso em: 19 maio 2016.

CELESTRINO, J. O.; COSTA, A. S. A prática de atividade física entre escolares com sobrepeso e obesidade. Revista Mackenzie de Educação Física e Esporte, São Paulo, v. 5, n. especial, p. 47-54, set./dez. 2006.

DIAS, I. B. F.; MONTENEGRO, R. A.; MONTEIRO, W. D. Exercício físico como estratégia de prevenção e tratamento da obesidade: aspectos fisiológicos e metodológicos. Revista Hospital Universitário Pedro Ernesto, Rio de Janeiro, v. 13, n. 1, p. 70-79, jan./mar. 2014. doi:10.12957/rhupe.2014.9808
ENNIS, C. D. Reimagining professional competence in physical education. Motriz Revista de Educação Física, Rio Claro, v. 19, n. 4, p. 662-672, out./dec. 2013. doi: 10.1590/S1980-65742013000400001

ESMERALDO, R. S. et al. Prevenção da obesidade de forma multidisciplinar no contexto escolar. Universitas: Ciências da Saúde, Brasília, v. 12, n. 1, p. 59-67, jan./jun. 2014. doi: 10.5102/ucs.v12i1.2682

GUEDES, D. P.; GUEDES, J. E. R. P. Esforços físicos nos programas de Educação Física Escolar. Revista Paulista de Educação Física, São Paulo, v. 15, n. 2, p. 33-44, jan./ jun. 2001.

GUIMARÃES, R. F. et al. Association between sedentary behavior and anthropometric and metabolic profiles among adolescents. Motriz Revista de Educação Física, Rio Claro, v. 19, n. 4, p. 753-762, out./dez. 2013. doi: 10.1590/S1980-65742013000400013

KWON, S. et al. Active lifestyle in childhood and adolescence prevents obesity develepment in young aldulthood. Pediatric Obesity, Malden, v. 23, n. 12, p. 2462-2469, Dec. 2015. doi:10.1002/oby.21262

LAWLOR, D. A. et al. Association between general and central adiposity in childhood, and changes in these, with cardiovascular risk factors in adolescence: prospective cohort study. British Medical Journal, London, v. 341, p. c6224, nov. 2010. http://dx.doi.org/10.1136/bmj. c6224

LEÃO, L. S. C. S. et al. Prevalência de obesidade em escolares de Salvador, Bahia. Arquivos Brasileiros de Endocrinologia e Metabologia, São Paulo, v. 47 , n. 2, p. 151-157, abr. 2003. doi:10.1590/S000427302003000200007

NASCIMENTO, L. A. A percepção do professor de Educação Física na prevenção da obesidade infantil. 2013. 69 f. Trabalho de Conclusão de Curso (Graduação) Universidade de Brasília, Brasília, 2013.

ONIS, M. et.al. Development of a WHO growth reference for school-aged children and adolescents. Geneva: World Health Organization, 2007.

RAMOS, M. L. M. et al. Sobrepeso e obesidade em escolares de 10 a 14 anos. Revista Brasileira de Promoção de Saúde, Fortaleza, v. 26, n. 2, p. 223-232, abr./jun. 2013. doi:10.5020/18061230.2013.p223

RICARDO, G. D.; CALDEIRA, G. V.; CORSO, A. C. T. Prevalência de sobrepeso e obesidade e indicadores de adiposidade central em escolares de Santa Carina, Brasil. Revista Brasileira de Epidemiologia, São Paulo, v. 12 , n. 3 , p. $425-435$, jun. 2009 . doi: 10.1590/S1415$790 X 2009000300011$. 
SACCHETTI, R. et al. Effects of a 2 year school-based interventoin of enhanced physical education in the primary school. The Journal of School Health, Columbus, v. 83, n. 9, p. 639-646, set. 2013. doi: 10.1111/josh.12076

SACCHETTI, R. et. al. Effect of a school based intervention to promote healthy habits in children 8-11 years old, living in the lowland area of Bologna Local Health Unit. Annali di Igiene: Medicina Preventiva e di Comunità, Roma, v. 27, n. 2, p. 432-446, jun. 2015. doi:10.7416/ ai. 2015.2030

SANTOS, A. L.; CARVALHO, A. L.; GARCIA JÚNIOR, J. R. Obesidade infantil e uma proposta de Educação Física preventiva. Motriz Revista de Educação Física, Rio Claro, v. 13, n. 3, p. 203-213, jul./set. 2007.
SOCIEDADE BRASILEIRA DE CARDIOLOGIA. I Diretriz de Prevenção da Aterosclerose na Infância e na Adolescência (versão final). Arquivos Brasileiros de Cardiologia, Rio de Janeiro, v. 85, n. Suppl 6, p. 1-36. dez. 2005. doi: 10.1590/S0066-782X2005002500001

WOLFF-HUGHES, D. L. et al. Population-referenced percentiles for waist-worn accelerometer-derived total activity counts in U.S. Youth: 2003 - 2006 NHANES. PLoS One, San Francisco, v. 9, n. 12, p. e115915, Dec. 2014. doi: 10.1371/journal.pone.0115915

ZANCHA, D. et al. Conhecimento dos professores de Educação Física Escolar sobre a abordagem Saúde Renovada e a temática saúde. Revista da Faculdade de Educação Física da UNICAMP, Campinas, v. 11, n. 1, p. 204217, jan./mar. 2013. doi:10.20396/conex.v11i1.8637638 